\title{
Erratum to: Mental Health and Its Associated Variables Among International Students at a Japanese University: With Special Reference to Their Financial Status
}

\author{
Kumi Kono $^{1}$ - Sharareh Eskandarieh ${ }^{2}$ Yoshihide Obayashi ${ }^{1} \cdot$ Asuna Arai $^{1}$ • \\ Hiko Tamashiro ${ }^{1}$
}

Published online: 11 August 2015

(C) Springer Science+Business Media New York 2015

\section{Erratum to: J Immigrant Minority Health DOI 10.1007/s10903-014-0100-1}

In the original version of this article, one of the five author names was listed incorrectly as "Sharareh Eskanadrieh." The name should be listed as: "Sharareh Eskandarieh." It is corrected with this erratum.

The online version of the original article can be found under doi: 10.1007/s10903-014-0100-1.

Asuna Arai

asunar@med.hokudai.ac.jp

1 Department of Global Health and Epidemiology, Hokkaido University Graduate School of Medicine, Kita 15, Nishi 7,

Kita-ku, Sapporo, Hokkaido 060-8638, Japan

2 Division of Mental Health and Social Health, and Addiction Prevention and Treatment, Center for Disease Control, Ministry of Health and Medical Education, Tehran, Iran 